\title{
On Eisenstein series with characters and Dedekind sums
}

\author{
by \\ Chizuru Sekine (Tokyo)
}

1. Introduction. Dedekind sums with characters were first introduced by Berndt. He basically considered a primitive character and defined some kinds of sums. Each sum arose in the transformation formulas of Eisenstein series with character, and Berndt obtained the reciprocity law for them by combining those transformation formulas (cf. $[2,3])$.

In [4], for coprime positive integers $k$ and $h$, and Dirichlet characters $\chi_{1} \bmod k$ and $\chi_{2} \bmod h$, respectively, we defined Dedekind sum $s_{n}\left(\left(\chi_{1}, k\right)\right.$, $\left.\left(\chi_{2}, h\right)\right)$ by

$$
s_{n}\left(\left(\chi_{1}, k\right),\left(\chi_{2}, h\right)\right)=k^{n-1} \sum_{a=0}^{k-1} \sum_{b=0}^{h-1} \frac{b}{h} \chi_{1}(a) \chi_{2}(b) \bar{B}_{n}\left(\frac{a}{k}+\frac{b}{h}\right),
$$

and obtained the reciprocity law. The proof of the law was given by deforming Barnes's double zeta function with a character into single zeta functions (Dirichlet $L$-functions), where the sums appeared as the difference between them. When $n=1$, our law is the following:

Theorem A. For $\chi_{1}$ and $\chi_{2}$ both non-trivial,

$$
\begin{aligned}
s_{1}\left(\left(\chi_{1}, k\right),\left(\chi_{2}, h\right)\right) & +s_{1}\left(\left(\chi_{2}, h\right),\left(\chi_{1}, k\right)\right) \\
= & \frac{B_{2, \chi_{1} \chi_{2}}}{2 h k \chi_{1}(h) \chi_{2}(k)}+\frac{B_{1, \chi_{1} \chi_{2}}}{\chi_{1}(h) \chi_{2}(k)}+B_{1, \chi_{1}} B_{1, \chi_{2}} .
\end{aligned}
$$

This theorem is applied to the calculation of class numbers of imaginary quadratic fields such as giving another proof of the class number formulae of Lerch and Mordell (cf. [5]).

When $\chi_{2}$ is the trivial character $\chi_{0}$, our sums $s_{1}\left(\left(\chi_{0}, h\right),\left(\chi_{1}, k\right)\right)$ and $s_{1}\left(\left(\chi_{1}, k\right),\left(\chi_{0}, h\right)\right)$ almost correspond to Berndt's sums $S_{1}\left(h, k ; \chi_{1}\right)$ and $S_{2}\left(k, h ; \bar{\chi}_{1}\right)$ defined in [3], respectively, and Theorem A becomes essentially the same as one of the two reciprocity laws between $S_{1}$ and $S_{2}$. Therefore

2000 Mathematics Subject Classification: 11F20, 11M36. 
we can say that our sums and the law are generalized cases of his sums $S_{1}$ and $S_{2}$.

In this paper, following Berndt's method, first we show that our sums for $n=1$ with non-principal primitive characters $\chi_{1}$ and $\chi_{2}$ also appear in the transformation formulas of Eisenstein series just as $S_{1}$ and $S_{2}$ did, and then give another proof of Theorem A by combining those transformation formulas. There we need two kinds of Eisenstein series with $\chi_{1}$ and $\chi_{2}$ : the series $G_{1}\left(z, s ; \chi_{1} \chi_{2} ; r_{1}, r_{2}\right)$ used by Berndt (see Section 2 ) and new Eisenstein series $G\left(z, s ; \chi_{1}, \chi_{2} ; r_{1}, r_{2}\right)$ slightly modified from $G_{1}$ (Definition 3.1). Here we take $\chi_{1} \chi_{2}$ instead of $\chi_{1}$ in $G_{1}$, and the transformation formula of $G_{1}$ is somewhat different from those of Berndt in [3].

In Section 2, we explain notations and give some preliminary results that we will need to prove Theorem A in Section 3.

2. Notations and preliminary results. We follow Berndt's notation. Set $\mathscr{H}=\{z \in \mathbb{C} \mid \operatorname{Im}(z)>0\}$ and $\sigma=\operatorname{Re}(s)$. We write $e(z)$ for $e^{2 \pi i z}$. As usual, $\{x\}$ denotes the fractional part of $x$, and $[x]$ denotes the greatest integer not exceeding $x$. Set $V(z)=V z=\frac{a z+b}{c z+d}$, where $a, b, c$, and $d$ are integers with $c>0$ and $a d-b c=1$. Unless otherwise stated, choose the branch of $\log z$ with $-\pi \leq \arg z<\pi$. In this section, let $\chi$ denote a primitive character $\bmod k$.

Let

$$
G(z, \chi)=\sum_{a=1}^{k-1} \chi(a) e\left(\frac{a z}{k}\right)
$$

denote the classical Gauss sum and put $G(\chi)=G(1, \chi)$.

For $z \in \mathscr{H}$ and $\sigma>1$, we have the Lipschitz summation formula ([3, $(2.2)])$

$$
\sum_{n=-\infty}^{\infty}(n+z)^{-s}=\frac{(-2 \pi i)^{s}}{\Gamma(s)} \sum_{n=1}^{\infty} e(n z) n^{s-1}
$$

and the character analogue of the Lipschitz summation formula $([3,(2.3)])$

$$
\sum_{n=-\infty}^{\infty} \chi(n)(n+z)^{-s}=\frac{G(\chi)(-2 \pi i / k)^{s}}{\Gamma(s)} \sum_{n=1}^{\infty} \bar{\chi}(n) e(n z / k) n^{s-1}
$$

The Bernoulli polynomials $B_{n}(x)$ and the Bernoulli functions $\bar{B}_{n}(x)$ are defined by

$$
\frac{t e^{x t}}{e^{t}-1}=\sum_{n=0}^{\infty} \frac{B_{n}(x)}{n !}
$$


and

$$
\bar{B}_{n}(x)=B_{n}(\{x\}) \quad \text { if } n>1, \quad \bar{B}_{1}(x)= \begin{cases}B_{1}(\{x\}) & \text { if } x \notin \mathbb{Z}, \\ 0 & \text { if } x \in \mathbb{Z}\end{cases}
$$

respectively. Also we define the generalized Bernoulli numbers $B_{n, \chi}$ by

$$
\sum_{a=1}^{k} \frac{\chi(a) t e^{a t}}{e^{k t}-1}=\sum_{n=0}^{\infty} B_{n, \chi} \frac{t^{n}}{n !}
$$

and then

$$
B_{n, \chi}=k^{n-1} \sum_{a=1}^{k} \chi(a) B_{n}\left(\frac{a}{k}\right)
$$

Then Dedekind sums defined by Berndt and the reciprocity law are the following:

DEFINITION 2.1 ([3, p. 314]). Let $\chi$ be an even primitive character of modulus $k$, and $c$ and $d$ be coprime positive integers. Set

$$
\begin{aligned}
& S_{1}(d, c ; \chi)=\sum_{n \bmod c k} \chi(n) \bar{B}_{1}\left(\frac{n}{c k}\right) \bar{B}_{1}\left(\frac{d n}{c}\right), \\
& S_{2}(d, c ; \chi)=\sum_{n \bmod c k} \bar{B}_{1}\left(\frac{n}{c k}\right) \sum_{a=1}^{k-1} \bar{\chi}(a) \bar{B}_{1}\left(\frac{d n / c+a}{k}\right) .
\end{aligned}
$$

TheOREM $2.2([3$, p. 318]). When $c \equiv 0(\bmod k)$,

$$
S_{1}(d, c ; \chi)+S_{2}(c, d ; \bar{\chi})=\left(\frac{d}{2 c}+\frac{\bar{\chi}(d)}{2 c d}\right) B_{2, \chi} .
$$

Theorem 2.2 also holds when $\chi$ is odd, since $S_{i}(d, c ; \chi)=0$ for $i=$ 1,2 , and $B_{2, \chi}=0$ (cf. [1]). To prove the above theorem, Berndt used the transformation formula of the following Eisenstein series.

Definition 2.3 ([3, p. 306]). For a primitive character $\chi \bmod k$, real numbers $r_{1}$ and $r_{2}$, a complex number $s$ with $\operatorname{Re}(s)>2$, and $z$ in $\mathscr{H}$, we define

$$
G_{1}\left(z, s ; \chi ; r_{1}, r_{2}\right)=\sum_{m, n=-\infty}^{\infty} \frac{\chi(m)}{\left(\left(m+r_{1}\right) z+n+r_{2}\right)^{s}},
$$

where $\sum^{\prime}$ means that the pair $(m, n)=\left(-r_{1},-r_{2}\right)$ is omitted if $r_{1}$ and $r_{2}$ are both integers.

3. Proof of the reciprocity law when $\chi=\chi_{1} \chi_{2}$. From now on, we assume that both $\chi_{1}$ and $\chi_{2}$ are primitive and non-trivial. First we introduce the new Eisenstein series defined below. 
DeFinition 3.1. Let $r_{1}$ and $r_{2}$ be arbitrary real numbers. For $\sigma>2$ and $z \in \mathscr{H}$, define

$$
G\left(z, s ; \chi_{1}, \chi_{2} ; r_{1}, r_{2}\right)=\sum_{m, n=-\infty}^{\infty} \frac{\chi_{1}(m) \chi_{2}(n)}{\left(\left(m+r_{1}\right) z+n+r_{2}\right)^{s}},
$$

where $\sum^{\prime}$ has the same meaning as in (2.4). Also for $z \in \mathscr{H}$ and $s \in \mathbb{C}$, set

$$
A\left(z, s ; \chi_{1}, \chi_{2} ; r_{1}, r_{2}\right)=\sum_{m>-r_{1}} \chi_{1}(m) \sum_{n=1}^{\infty} \chi_{2}(n) e\left(n\left(\left(m+r_{1}\right) z+r_{2}\right) / h\right) n^{s-1}
$$

and

$$
\begin{aligned}
& H\left(z, s ; \chi_{1}, \chi_{2} ; r_{1}, r_{2}\right) \\
& \quad=A\left(z, s ; \chi_{1}, \chi_{2} ; r_{1}, r_{2}\right)+\chi_{1}(-1) \chi_{2}(-1) e(s / 2) A\left(z, s ; \chi_{1}, \chi_{2} ;-r_{1},-r_{2}\right) .
\end{aligned}
$$

We note that Berndt also defined $G, A$, and $H$ in [3, p. 302]. He assumed that both $\chi_{1}$ and $\chi_{2}$ are primitive characters $\bmod k$.

For $\sigma>0$ and $a$ real, let

$$
L\left(s, \chi_{2}, a\right)=\sum_{n>-a} \chi_{2}(n)(n+a)^{-s}
$$

which is analytically continued to an entire function of $s$, and let

$$
\mathscr{L}_{ \pm}\left(s, \chi_{2}, a\right)=L\left(s, \chi_{2}, a\right)+\chi_{2}(-1) e( \pm s / 2) L\left(s, \chi_{2},-a\right) .
$$

Then the following proposition holds.

Proposition 3.2. For $\sigma>2$ and $z \in \mathscr{H}$,

$$
\begin{aligned}
& G\left(z, s ; \chi_{1}, \chi_{2} ; r_{1}, r_{2}\right) \\
& \quad=\frac{G\left(\chi_{2}\right)(-2 \pi i / h)^{s}}{\Gamma(s)} H\left(z, s ; \chi_{1}, \bar{\chi}_{2} ; r_{1}, r_{2}\right)+\chi_{1}\left(-r_{1}\right) \mathscr{L}_{+}\left(s, \chi_{2}, r_{2}\right) .
\end{aligned}
$$

Proof. The proof goes as in [2, pp. 12, 13]. We put

$$
\begin{aligned}
G\left(z, s ; \chi_{1}, \chi_{2} ; r_{1}, r_{2}\right) & =\chi_{1}\left(-r_{1}\right) \sum_{n=-\infty}^{\infty} \frac{\chi_{2}(n)}{\left(n+r_{2}\right)^{s}} \\
& +\left(\sum_{m<-r_{1}} \sum_{n=-\infty}^{\infty}+\sum_{m>-r_{1}} \sum_{n=-\infty}^{\infty}\right) \frac{\chi_{1}(m) \chi_{2}(n)}{\left(\left(m+r_{1}\right) z+n+r_{2}\right)^{s}} \\
= & S_{1}+S_{2}+S_{3},
\end{aligned}
$$

where $\sum^{\prime \prime}$ means that $n=-r_{2}$ is omitted if $r_{2}$ is an integer. 
Firstly,

$$
\begin{aligned}
S_{1} & =\chi_{1}\left(-r_{1}\right)\left(\sum_{n>-r_{2}} \chi_{2}(n)\left(n+r_{2}\right)^{-s}+\sum_{n>r_{2}} \chi_{2}(-n)\left(-n+r_{2}\right)^{-s}\right) \\
& =\chi_{1}\left(-r_{1}\right)\left(L\left(s, \chi_{2}, r_{2}\right)+\chi_{2}(-1) e(s / 2) L\left(s, \chi_{2},-r_{2}\right)\right) .
\end{aligned}
$$

Next, by replacing $n$ by $-n, m$ by $-m$, and using (2.2),

$$
\begin{aligned}
S_{2} & =\chi_{1} \chi_{2}(-1) e(s / 2) \sum_{m>r_{1}} \chi_{1}(m) \sum_{n=-\infty}^{\infty} \chi_{2}(n)\left(\left(m-r_{1}\right) z+n-r_{2}\right)^{-s} \\
& =\chi_{1} \chi_{2}(-1) e(s / 2) \frac{G\left(\chi_{2}\right)(-2 \pi i / h)^{s}}{\Gamma(s)} A\left(z, s ; \chi_{1}, \bar{\chi}_{2},-r_{1},-r_{2}\right) .
\end{aligned}
$$

Lastly, by using (2.2),

$$
S_{3}=\frac{G\left(\chi_{2}\right)(-2 \pi i / h)^{s}}{\Gamma(s)} A\left(z, s ; \chi_{1}, \bar{\chi}_{2}, r_{1}, r_{2}\right) .
$$

Combining these three, (3.2) is obtained.

Definition 3.3. Let $Q=\{z=x+i y: x>-d / c, y>0\}$, and set $R_{1}=a r_{1}+c r_{2}, R_{2}=b r_{1}+d r_{2}$, and $\varrho=\left\{R_{2}\right\} c-\left\{R_{1}\right\} d$, where $a, b, c$ and $d$ are integers with $c>0$ and $a d-b c=1$. For non-negative integers $j, \mu, \nu$, positive integers $h$ and $k$, and for $z \in Q$, let

$$
f\left(z, s, r_{1}, r_{2}, j, \mu, \nu, h, k\right)=\int_{C} u^{s-1} \frac{e^{-\frac{c \mu+j-\left\{R_{1}\right\}}{c h}(c z+d) h u}}{e^{-(c z+d) h u}-1} \cdot \frac{e^{\frac{\nu+\left\{\frac{\underline{\rho}+d j}{c}\right\}}{k} k u}}{e^{k u}-1} d u
$$

where we choose the branch of $u^{s}$ with $0<\arg u<2 \pi$, and $C$ is a loop beginning at $+\infty$, proceeding in $\mathscr{H}$, encircling the origin in the positive direction, and then returning to $+\infty$ in the lower half-plane.

When $s=-N$, where $N$ is a non-negative integer, a simple application of Cauchy's residue theorem yields

$$
\begin{aligned}
f(z,-N, & \left.r_{1}, r_{2}, j, \mu, \nu, h, k\right) \\
= & 2 \pi i \sum_{m+n=N+2} B_{m}\left(\frac{c \mu+j-\left\{R_{1}\right\}}{c h}\right) B_{n}\left(\frac{\nu+\{(d j+\varrho) / c\}}{k}\right) \\
& \times \frac{(-(c z+d) h)^{m-1} k^{n-1}}{m ! n !} .
\end{aligned}
$$

Then we have the transformation formulas for $H$, or equivalently for $G$.

Theorem 3.4. Suppose that $a \equiv 0(\bmod h)$ and $d \equiv 0(\bmod k)$. Then for $z \in Q$ and $s \in \mathbb{C}$,

$$
\begin{aligned}
(c z+d)^{-s}(-2 \pi i / h)^{s} G\left(\chi_{2}\right) H\left(V z, s ; \chi_{1}, \bar{\chi}_{2} ; r_{1}, r_{2}\right) & \\
& +\chi_{1}\left(-r_{1}\right)(c z+d)^{-s} \Gamma(s) \mathscr{L}_{+}\left(s, \chi_{2}, r_{2}\right)
\end{aligned}
$$




$$
\begin{aligned}
= & \chi_{1}(-c) \chi_{2}(-b)\left((-2 \pi i / k)^{s} G\left(\chi_{1}\right) H\left(z, s ; \chi_{2}, \bar{\chi}_{1} ; R_{1}, R_{2}\right)\right. \\
& +\chi_{2}\left(-R_{1}\right) \Gamma(s) \mathscr{L}_{-}\left(s, \chi_{1}, R_{2}\right) \\
& +\chi_{1} \chi_{2}(-1) e(-s / 2) \\
& \times \sum_{j=1}^{c} \sum_{\mu=0}^{h-1} \sum_{\nu=0}^{k-1} \chi_{2}\left(c \mu+j+\left[R_{1}\right]\right) \chi_{1}\left(-\nu+\left[R_{2}+d\left(j-\left\{R_{1}\right\}\right) / c\right]\right) \\
& \left.\times f\left(z, s, r_{1}, r_{2}, j, \mu, \nu, h, k\right)\right) .
\end{aligned}
$$

Here $V z=\frac{a z+b}{c z+d}$ with integers $a, b, c$, and $d$ satisfying $c>0$ and $a d-b c=1$, and we use the convention that $\chi(x)=0$ when $\chi$ is a character and $x$ is a non-integer.

Proof. The proof is along the same lines as that of Theorem 2 in [2]. For $z \in \mathscr{H}$ and $\sigma>2$,

$$
G\left(V z, s ; \chi_{1}, \chi_{2} ; r_{1}, r_{2}\right)=\sum_{m, n=-\infty}^{\infty} \chi_{1}(m) \chi_{2}(n)\left(\frac{\left(M+R_{1}\right) z+N+R_{2}}{c z+d}\right)^{-s}
$$

where $M=m a+n c$ and $N=m b+n d$. As the pair $(m, n)$ ranges over all pairs of integers except possibly $\left(-r_{1},-r_{2}\right)$, it follows that $(M, N)$ ranges over all pairs of integers except possibly $\left(-R_{1},-R_{2}\right)$. Therefore when $a \equiv 0$ $(\bmod h)$ and $d \equiv 0(\bmod k)$,

$$
\begin{aligned}
G\left(V z, s ; \chi_{1}, \chi_{2} ; r_{1}, r_{2}\right) & \\
& =\sum_{M, N=-\infty}^{\infty} \chi_{1}(M d-N c) \chi_{2}(N a-M b)\left(\frac{\left(M+R_{1}\right) z+N+R_{2}}{c z+d}\right)^{-s} \\
& =\chi_{1}(-c) \chi_{2}(-b) \sum_{m, n=-\infty}^{\infty} \chi_{1}(n) \chi_{2}(m)\left(\frac{\left(m+R_{1}\right) z+n+R_{2}}{c z+d}\right)^{-s} .
\end{aligned}
$$

Now we use the following lemma.

Lemma 3.5 ([2, p. 13]). Let $A, B, C$ and $D$ be real with $A$ and $B$ not both zero and $C>0$. Then for $z \in \mathscr{H}$,

$$
\arg ((A z+B) /(C z+D))=\arg (A z+B)-\arg (C z+D)+2 \pi k,
$$

where $k$ is independent of $z \in \mathscr{H}$, and $k=1$ if $A \leq 0$ and $A D-B C>0$, and 0 otherwise.

We go back to the proof of the theorem. From the above lemma,

$$
(c z+d)^{-s} G\left(V z, s ; \chi_{1}, \chi_{2} ; r_{1}, r_{2}\right)
$$

$$
=\chi_{1}(-c) \chi_{2}(-b)\left(e(-s) \sum_{\substack{m+R_{1} \leq 0 \\ d\left(m+R_{1}\right)>c\left(n+R_{2}\right)}}+\sum_{\text {otherwise }}^{\prime}\right) \frac{\chi_{2}(m) \chi_{1}(n)}{\left(\left(m+R_{1}\right) z+n+R_{2}\right)^{s}}
$$




$$
\begin{aligned}
= & \chi_{1}(-c) \chi_{2}(-b) \\
& \times\left(G\left(z, s ; \chi_{2}, \chi_{1} ; R_{1}, R_{2}\right)+(e(-s)-1) g\left(z, s ; \chi_{2}, \chi_{1} ; R_{1}, R_{2}\right)\right),
\end{aligned}
$$

where

$$
g\left(z, s ; \chi_{2}, \chi_{1} ; R_{1}, R_{2}\right)=\sum_{\substack{m+R_{1} \leq 0 \\ d\left(m+R_{1}\right)>c\left(n+R_{2}\right)}} \frac{\chi_{2}(m) \chi_{1}(n)}{\left(\left(m+R_{1}\right) z+n+R_{2}\right)^{s}}
$$

Replacing $m$ by $-m$ and $n$ by $-n$ and separating the terms with $m=R_{1}$, we obtain

$$
\begin{aligned}
& g\left(z, s ; \chi_{2}, \chi_{1} ; R_{1}, R_{2}\right) \\
& \quad=e(s / 2) \chi_{1} \chi_{2}(-1)\left(\chi_{2}\left(R_{1}\right) L\left(s, \chi_{1},-R_{2}\right)+h\left(z, s ; \chi_{2}, \chi_{1} ; R_{1}, R_{2}\right)\right),
\end{aligned}
$$

where

$$
h\left(z, s ; \chi_{2}, \chi_{1} ; R_{1}, R_{2}\right)=\sum_{\substack{m>R_{1} \\ n>R_{2}+\frac{d}{c}\left(m-R_{1}\right)}} \frac{\chi_{2}(m) \chi_{1}(n)}{\left(\left(m-R_{1}\right) z+n-R_{2}\right)^{s}}
$$

In the double sum, $\operatorname{Re}\left(\left(m-R_{1}\right) z+n-R_{2}\right)>0$ if $x>-d / c$. Using Euler's integral representation of $\Gamma(s)$, we have for $z \in Q$ and $\sigma>2$,

$$
\begin{aligned}
& \Gamma(s) h\left(z, s ; \chi_{2}, \chi_{1} ; R_{1}, R_{2}\right) \\
& =\sum_{\substack{m>R_{1} \\
n>R_{2}+\frac{d}{c}\left(m-R_{1}\right)}} \chi_{2}(m) \chi_{1}(n) \int_{0}^{\infty} u^{s-1} \exp \left(-\left(m-R_{1}\right) z u-\left(n-R_{2}\right) u\right) d u .
\end{aligned}
$$

Put $m=m^{\prime} c+j+\left[R_{1}\right]+1,0 \leq m^{\prime}<\infty, 0 \leq j \leq c-1$ and $n=$ $n^{\prime}+\left[R_{2}+d\left(m-R_{1}\right) / c\right]+1,0 \leq n^{\prime}<\infty$. Since $d \equiv 0(\bmod k)$, we have

$$
\begin{aligned}
& \Gamma(s) h\left(z, s ; \chi_{2}, \chi_{1} ; R_{1}, R_{2}\right) \\
& =\sum_{j=0}^{c-1} \sum_{m^{\prime}=0}^{\infty} \sum_{n^{\prime}=0}^{\infty} \chi_{2}\left(m^{\prime} c+j+1+\left[R_{1}\right]\right) \chi_{1}\left(n^{\prime}+1+\left[R_{2}+d\left(j+1-\left\{R_{1}\right\}\right) / c\right]\right) \\
& \quad \times \int_{0}^{\infty} u^{s-1} \exp \left(-\left(m^{\prime} c+j+1-\left\{R_{1}\right\}\right) z u\right) \\
& \quad \times \exp \left(-\left(n^{\prime}+1+d m^{\prime}-R_{2}+\left[R_{2}+d\left(j+1-\left\{R_{1}\right\}\right) / c\right]\right) u\right) d u .
\end{aligned}
$$

Replacing $j+1$ by $j$, and putting $m^{\prime}=m h+\mu, 0 \leq m<\infty, 0 \leq \mu \leq h-1$, and $n^{\prime}=n k+\nu, 0 \leq n<\infty, 0 \leq \nu \leq k-1$, we obtain 


$$
\begin{aligned}
& \quad \Gamma(s) h\left(z, s ; \chi_{2}, \chi_{1} ; R_{1}, R_{2}\right) \\
& =\sum_{j=1}^{c} \sum_{\mu=0}^{h-1} \sum_{\nu=0}^{k-1} \chi_{2}\left(c \mu+j+\left[R_{1}\right]\right) \chi_{1}\left(\nu+1+\left[R_{2}+d\left(j-\left\{R_{1}\right\}\right) / c\right]\right) \\
& \times \int_{0}^{\infty} u^{s-1} \exp \left(-\left(c \mu+j-\left\{R_{1}\right\}\right) z u-\left(\nu+1+d \mu-R_{2}\right.\right. \\
& \times \sum_{m=0}^{\infty} \sum_{n=0}^{\infty} \exp (-m h c z u-m d h u-n k u) d u
\end{aligned}
$$$$
=\sum_{j=1}^{c} \sum_{\mu=0}^{h-1} \sum_{\nu=0}^{k-1} \chi_{2}\left(c \mu+j+\left[R_{1}\right]\right) \chi_{1}\left(\nu+1+\left[R_{2}+d\left(j-\left\{R_{1}\right\}\right) / c\right]\right)
$$$$
\times \int_{0}^{\infty} u^{s-1} \frac{\exp \left(-\left(\left(c \mu+j-\left\{R_{1}\right\}\right) / c h\right)(c z+d) h u\right)}{1-\exp (-(c z+d) h u)}
$$$$
\times \frac{\exp \left(\left(-\nu-1+d j / c-\left\{R_{1}\right\} d / c+R_{2}-\left[R_{2}+d\left(j-\left\{R_{1}\right\}\right) / c\right]\right) u\right)}{1-\exp (-k u)} d u
$$$$
=-\sum_{j=1}^{c} \sum_{\mu=0}^{h-1} \sum_{\nu=0}^{k-1} \chi_{2}\left(c \mu+j+\left[R_{1}\right]\right) \chi_{1}\left(-\nu+\left[R_{2}+d\left(j-\left\{R_{1}\right\}\right) / c\right]\right)
$$$$
\times \int_{0}^{\infty} u^{s-1} \frac{\exp \left(-\left(\left(c \mu+j-\left\{R_{1}\right\}\right) / c h\right)(c z+d) h u\right)}{\exp (-(c z+d) h u)-1}
$$$$
\times \frac{\exp (((\nu+\{(d j+\varrho) / c\}) / k) k u)}{\exp (k u)-1} d u
$$

$$
\begin{aligned}
=-\sum_{j=1}^{c} \sum_{\mu=0}^{h-1} \sum_{\nu=0}^{k-1} \chi_{2}\left(c \mu+j+\left[R_{1}\right]\right) \chi_{1}(-\nu+ & {\left.\left[R_{2}+d\left(j-\left\{R_{1}\right\}\right) / c\right]\right) } \\
\times & \frac{f\left(z, s, r_{1}, r_{2}, j, \mu, \nu, h, k\right)}{e(s)-1},
\end{aligned}
$$

where in the next-to-last equality we replaced $k-1-\nu$ by $\nu$. Combining (3.5)-(3.7), and using (3.2), we obtain (3.4).

For $G_{1}$ in (2.4), Berndt derived the following equation:

Proposition $3.6[3,(4.3)]$. For $\sigma>2$ and $z \in \mathscr{H}$,

$$
G_{1}\left(z, s ; \chi ; r_{1}, r_{2}\right)=\frac{(-2 \pi i)^{s}}{\Gamma(s)} H_{1}\left(z, s ; \chi ; r_{1}, r_{2}\right)+\chi\left(-r_{1}\right) Z_{+}\left(s, r_{2}\right),
$$


where

$$
\begin{gathered}
A_{1}\left(z, s ; \chi ; r_{1}, r_{2}\right)=\sum_{m>-r_{1}} \chi(m) \sum_{n=1}^{\infty} e\left(n\left(\left(m+r_{1}\right) z+r_{2}\right)\right) n^{s-1}, \\
H_{1}\left(z, s ; \chi ; r_{1}, r_{2}\right)=A_{1}\left(z, s ; \chi ; r_{1}, r_{2}\right)+\chi(-1) e(s / 2) A_{1}\left(z, s ; \chi ;-r_{1},-r_{2}\right), \\
Z(s, a)=\sum_{n>-a}(n+a)^{-s}, \quad Z_{+}(s, a)=Z(s, a)+e(s / 2) Z(s,-a) .
\end{gathered}
$$

Now we take $\chi=\chi_{1} \chi_{2}$ in (2.4) and obtain a new transformation formula for $G_{1}\left(z, s ; \chi_{1} \chi_{2} ; r_{1}, r_{2}\right)$.

TheOREM 3.7. (i) Suppose that $c \equiv 0(\bmod h)$ and $d \equiv 0(\bmod k)$. Then for $z \in Q$ and $s \in \mathbb{C}$,

$$
\begin{aligned}
(c z+d)^{-s}(-2 \pi i)^{s} H_{1}\left(V z, s ; \chi_{1} \chi_{2} ; r_{1}, r_{2}\right) & \quad+\chi_{1} \chi_{2}\left(-r_{1}\right)(c z+d)^{-s} \Gamma(s) Z_{+}\left(s, r_{2}\right) \\
= & \chi_{1}(-c) \chi_{2}(d)\left(G\left(\chi_{1}\right)(-2 \pi i / k)^{s} H\left(z, s ; \chi_{2}, \bar{\chi}_{1} ; R_{1}, R_{2}\right)\right. \\
& +\chi_{1}\left(-R_{1}\right) \Gamma(s) \mathscr{L}_{-}\left(s, \chi_{1}, R_{2}\right) \\
+ & \chi_{1} \chi_{2}(-1) e(-s / 2) \\
& \times \sum_{j=1}^{c} \sum_{\mu=0}^{h-1} \sum_{\nu=0}^{k-1} \chi_{2}\left(j+\left[R_{1}\right]\right) \chi_{1}\left(-\nu+\left[R_{2}+d\left(j-\left\{R_{1}\right\}\right) / c\right]\right) \\
& \left.\times f\left(z, s, r_{1}, r_{2}, j, \mu, \nu, h, k\right)\right) .
\end{aligned}
$$

(ii) Suppose that $c \equiv 0(\bmod k)$ and $d \equiv 0(\bmod h)$. Then for $z \in Q$ and $s \in \mathbb{C}$,

$$
\begin{aligned}
(c z+d)^{-s}(-2 \pi i)^{s} H_{1}\left(V z, s ; \chi_{1} \chi_{2} ; r_{1}, r_{2}\right) & \quad+\chi_{1} \chi_{2}\left(-r_{1}\right)(c z+d)^{-s} \Gamma(s) Z_{+}\left(s, r_{2}\right) \\
=\chi_{1}(d) \chi_{2}(-c)\left(G\left(\chi_{2}\right)(-2 \pi i / h)^{s} H\left(z, s ; \chi_{1}, \bar{\chi}_{2} ; R_{1}, R_{2}\right)\right. & \\
+ & \chi_{1}\left(-R_{1}\right) \Gamma(s) \mathscr{L}_{-}\left(s, \chi_{2}, R_{2}\right) \\
+ & \chi_{1} \chi_{2}(-1) e(-s / 2) \\
& \times \sum_{j=1}^{c} \sum_{\mu=0}^{h-1} \sum_{\nu=0}^{k-1} \chi_{1}\left(j+\left[R_{1}\right]\right) \chi_{2}\left(-\mu+\left[R_{2}+d\left(j-\left\{R_{1}\right\}\right) / c\right]\right) \\
& \left.\times f\left(z, s, r_{1}, r_{2}, j, \nu, \mu, k, h\right)\right) .
\end{aligned}
$$

Here $V z$ and the convention for a character $\chi$ is as in Theorem 3.4.

Proof. The proof goes as that of Theorem 3.4. 
Corollary 3.8. (i) If $c \equiv 0(\bmod h)$ and $d \equiv 0(\bmod k)$, then

$$
\begin{aligned}
& H_{1}\left(V z, 0 ; \chi_{1} \chi_{2} ; 0,0\right) \\
& =\chi_{1}(-c) \chi_{2}(d) G\left(\chi_{1}\right) H\left(z, 0 ; \chi_{2}, \bar{\chi}_{1} ; 0,0\right) \\
& \quad-\frac{k \pi i}{c z+d} \chi_{1}(-c) \chi_{2}(-d) \sum_{j=1}^{c} \sum_{\nu=0}^{k-1} \chi_{1}(\nu) \chi_{2}(j) \bar{B}_{2}\left(\frac{\nu+d j / c}{k}\right) \\
& \quad+2 \pi i \chi_{1}(-c) \chi_{2}(-d) \sum_{j=1}^{c} \sum_{\nu=0}^{k-1} \chi_{1}(\nu) \chi_{2}(j) \bar{B}_{1}\left(\frac{j}{c}\right) \bar{B}_{1}\left(\frac{\nu+d j / c}{k}\right) .
\end{aligned}
$$

(ii) If $c \equiv 0(\bmod k)$ and $d \equiv 0(\bmod h)$, then

$$
\begin{aligned}
& H_{1}\left(V z, 0 ; \chi_{1} \chi_{2} ; 0,0\right) \\
& =\chi_{1}(d) \chi_{2}(-c) G\left(\chi_{2}\right) H\left(z, 0 ; \chi_{1}, \bar{\chi}_{2} ; 0,0\right) \\
& \quad-\frac{h \pi i}{c z+d} \chi_{1}(-d) \chi_{2}(-c) \sum_{j=1}^{c} \sum_{\mu=0}^{h-1} \chi_{1}(j) \chi_{2}(\mu) \bar{B}_{2}\left(\frac{\mu+d j / c}{h}\right) \\
& \quad+2 \pi i \chi_{1}(-d) \chi_{2}(-c) \sum_{j=1}^{c} \sum_{\mu=0}^{h-1} \chi_{1}(j) \chi_{2}(\mu) \bar{B}_{1}\left(\frac{j}{c}\right) \bar{B}_{1}\left(\frac{\mu+d j / c}{h}\right) .
\end{aligned}
$$

(iii) If $a \equiv 0(\bmod h)$ and $d \equiv 0(\bmod k)$, then

$$
\begin{aligned}
& G\left(\chi_{2}\right) H\left(V z, 0 ; \chi_{1}, \bar{\chi}_{2} ; 0,0\right) \\
&=\chi_{1}(-c) \chi_{2}(-b) G\left(\chi_{1}\right) H\left(z, 0 ; \chi_{2}, \bar{\chi}_{1} ; 0,0\right) \\
&+2 \pi i \chi_{1}(-c) \chi_{2}(b) \\
& \quad \times \sum_{j=1}^{c} \sum_{\mu=0}^{h-1} \sum_{\nu=0}^{k-1} \chi_{1}(\nu) \chi_{2}(c \mu+j) \bar{B}_{1}\left(\frac{c \mu+j}{c h}\right) \bar{B}_{1}\left(\frac{\nu+d j / c}{k}\right) .
\end{aligned}
$$

Proof. Put $s=r_{1}=r_{2}=0$ in (3.9), (3.10) and (3.4), respectively, and use (3.3).

Proof of Theorem A. Let $V^{*} z=(b z-a) /(d z-c)$ and set $c=k$ and $d=h$. Apply (3.11) to $V^{*}$. Then

$$
\begin{aligned}
& H_{1}\left(V^{*} z, 0 ; \chi_{1} \chi_{2} ; 0,0\right) \\
& =\chi_{1}(-h) \chi_{2}(-k) G\left(\chi_{1}\right) H\left(z, 0 ; \chi_{2}, \bar{\chi}_{1} ; 0,0\right)-\frac{\pi i \chi_{1} \chi_{2}(-1)}{h(h z-k)} B_{2, \chi_{1} \chi_{2}} \\
& \quad+\pi i \chi_{1} \chi_{2}(-1) B_{1, \chi_{1} \chi_{2}}-2 \pi i \chi_{1}(-h) \chi_{2}(-k) s_{1}\left(\left(\chi_{1}, k\right),\left(\chi_{2}, h\right)\right),
\end{aligned}
$$


where we used the fact that

$\left\{\left\{\frac{h \nu+k \mu}{k h}\right\} \mid \nu, \mu \in \mathbb{Z}, 0 \leq \nu<k, 0 \leq \mu<h\right\}=\left\{\frac{i}{k h} \mid 0 \leq i \leq h k-1\right\}$, and $\bar{B}_{1}(-\{x\})=B_{1}(1-\{x\})=-\{x\}+1 / 2$ for $x \notin \mathbb{Z}$, and (2.3).

Applying (3.12) with $z$ replaced by $-1 / z$, we have

$$
\begin{aligned}
& H_{1}\left(V^{*} z, 0 ; \chi_{1} \chi_{2} ; 0,0\right) \\
= & \chi_{1}(h) \chi_{2}(-k) G\left(\chi_{2}\right) H\left(-\frac{1}{z}, 0 ; \chi_{1}, \bar{\chi}_{2} ; 0,0\right)-\frac{\pi i z \chi_{1} \chi_{2}(-1)}{k(h z-k)} B_{2, \chi_{1} \chi_{2}} \\
& -\pi i \chi_{1} \chi_{2}(-1) B_{1, \chi_{1} \chi_{2}}+2 \pi i \chi_{1}(-h) \chi_{2}(-k) s_{1}\left(\left(\chi_{2}, h\right),\left(\chi_{1}, k\right)\right) .
\end{aligned}
$$

Lastly, taking $a=d=0, c=1$, and $b=-1$ in (3.13), we get

$$
\begin{aligned}
& G\left(\chi_{2}\right) H\left(-\frac{1}{z}, 0 ; \chi_{1}, \bar{\chi}_{2} ; 0,0\right) \\
& \quad=\chi_{1}(-1) G\left(\chi_{1}\right) H\left(z, 0 ; \chi_{2}, \bar{\chi}_{1} ; 0,0\right)-2 \pi i \chi_{1}(-1) B_{1, \chi_{1}} B_{1, \chi_{2}},
\end{aligned}
$$

where we used $B_{1, \chi_{2}}=-\chi_{2}(-1) B_{1, \chi_{2}}$. By subtracting (3.14) from (3.15), combining with (3.16), and dividing both sides by $2 \pi i \chi_{1}(-h) \chi_{2}(-k)$, we obtain (1.2).

Acknowledgements. The author thanks Prof. K. Ota for helpful advice about this paper.

\section{References}

[1] B. C. Berndt, Character analogues of the Poisson and Euler-MacLaurin summation formulas with applications, J. Number Theory 7 (1975), 413-445.

[2] - Character transformation formulae similar to those for the Dedekind eta-function, in: Proc. Sympos. Pure Math. 24, Amer. Math. Soc., Providence, RI, 1973, 9-30.

[3] -, On Eisenstein series with characters and the values of Dirichlet L-functions, Acta Arith. 28 (1975), 299-320.

[4] Y. Nagasaka, K. Ota and C. Sekine, Generalizations of Dedekind sums and their reciprocity laws, ibid. 106 (2003), 355-378.

[5] K. Ota, Dedekind sums with characters and class numbers of imaginary quadratic fields, ibid. 108 (2003), 203-215.

Department of Mathematics \& Computer Science

Tsuda College

Kodaira, Tokyo, 187-8577 Japan

E-mail: m99sekin@tsuda.ac.jp 